\title{
ENTRE LA POÉSIE ET LE TERRORISME : \\ LA GRANDE TRIBU : C'EST LA FAUTE À PAPINEAU \\ DE VICTOR-LÉVY BEAULIEU
}

VIEN, MYRIAM.

Candidate à la maîtrise en littérature, Université McGill

Résumé : Dans cet article, l'auteure se propose d'interpréter le projet révolutionnaire embrassé par La Grande tribu : c'est la faute à Papineau! de Victor-Lévy Beaulieu à la faveur de la notion de «terrorisme », un parti pris pour la radicalité qui s'incarne autant dans le régime politique mis en œuvre par les personnages que sur le plan langagier, à travers le mode d'expression contestataire célébré par le texte. En effet, valorisant la poésie automatiste de Claude Gauvreau, La Grande tribu pousse l'expérimentation langagière aux limites du consensus expressif et poursuit une logique radicale qui conclut à l'impuissance des mots et de la littérature. Ce faisant, en décortiquant la structure hybride de l'œuvre et l'intertexte gauvrien, le présent article tâche de révéler comment ces éléments convergent à inscrire la lutte et la rébellion au cœur du récit, au point d'envisager l'action terroriste comme ultime solution au malaise national.

Mots-clés : La Grande tribu : c'est à la faute à Papineau!, Victor-Lévy Beaulieu, terrorisme, radicalité, indépendance du Québec

\begin{abstract}
In this paper, the author interprets the revolutionary project comprehended in La Grande tribu : c'est la faute à Papineau! by Victor-Lévy Beaulieu, making use of the notion of "terrorism" as firm stance toward a radicalism equally embodied in the political regime implemented by the characters as well in the language, through an antiestablishment mode of expression celebrated by the text. Indeed, bringing out the Automatist poetry of Claude Gauvreau, La Grande tribu pushes linguistic experimentation to the limits of common expression and follows a radical logic that leads to the powerlessness of words and literature. In so doing, by decorticating the hybrid structure of the text and of the Gauvrian intertext, this paper seeks to reveal how these elements come together to inscribe struggle and rebellion at the heart of the narrative, to a point where terrorist action becomes the ultimate solution to national unease.
\end{abstract}

Keywords : La Grande tribu: c'est la faute à Papineau!, Victor-Lévy Beaulieu, terrorism, radicalism, Quebec independence 
L'écrivain et polémiste Victor-Lévy Beaulieu (VLB) engage toute son œuvre et son travail d'éditeur dans la cause de la souveraineté du Québec, faisant sien le mot du poète William Butler Yeats : « II n'y a pas de nationalité sans littérature, pas de littérature sans nationalité » (Beaulieu, 2006 : 116). C'est en menaçant de procéder à l'autodafé de son entière production que VLB brûle symboliquement son $70^{\circ}$ ouvrage, La Grande Tribu : c'est la faute à Papineau, le jour de son lancement, accusant le recul du mouvement indépendantiste. II confie alors à une journaliste présente lors de la scène : " Il faut être radical sinon il ne se passerait rien. Je pense qu'on a été molassons trop longtemps » (Guy, 2008). Ainsi sacrifiée sur l'autel du politique, La Grande Tribu est une œuvre sans nuances qui célèbre l'échec de la littérature à opérer un réel changement social. En vertu du principe sartrien, placé en épigraphe de l'œuvre, selon lequel « [on] n'est jamais assez radical » (Beaulieu, 2008 : 9), le récit envisage l'action terroriste comme ultime solution au malaise national, un terrorisme qui s'affiche tant dans les actions des personnages que sur le plan langagier, notamment par la mise en valeur dans le texte du mode d'expression poétique de Claude Gauvreau, l'exploréen, une langue éclatée et déstructurée qui pose obstacle à sa propre lisibilité. La Grande Tribu, en valorisant l'expérimentation langagière d'une poésie poussée aux limites du consensus expressif, poursuit une logique sans compromis qui conclut à l'impuissance des mots et de la littérature et opte résolument pour l'action radicale.

En avançant l'idée que la pratique littéraire de VLB s'affiche dans l'esprit d'une démarche «terroriste », nous détaillerons le projet derrière La Grande Tribu afin d'en dégager les éléments qui convergent à inscrire la lutte et la contestation au cœur du récit. En effet, soutenue par l'ambition véelbienne de conférer au projet une envergure épique, La Grande Tribu déploie une structure hybride en deux volets qui détermine un programme révolutionnaire et façonne la figure héroïque qui en constitue le porte-étendard. Celle-ci s'incarne à travers le personnage de l'orignal épormyable, une représentation dans l'œuvre du poète québécois Claude Gauvreau, dont l'œuvre dramaturgique et poétique, intertexte immanquable dans $L a$ Grande Tribu, sert d'illustration à la radicalité de sa démarche créatrice. Sa poésie automatiste, surtout, retenue comme un mode d'expression contestataire, échoue à créer un dialogue avec la société du texte, inapte à vivre dans la surréalité que convoque ce langage. Devant cette défaite du sens, c'est le terrorisme, matérialisé à la fin du récit, qui parvient à exécuter le renversement d'un système corrompu, devant lequel l'art poétique de l'orignal épormyable, incompris et méprisé, était sans recours.

Dès le début de sa carrière, VLB annonce la venue d'un «Livre totalisant », une œuvre fondatrice d'une mythologie proprement québécoise, une sorte de genèse dans laquelle le peuple québécois reconnaîtrait son histoire et sa vérité. Cet ouvrage, ce devait être La Grande Tribu : c'est la faute à Papineau, en chantier depuis le début des années 1980, un projet conçu à l'origine par VLB comme un grand récit historique retraçant le parcours des premiers colons français établis sur le territoire. Appelée à devenir une épopée nationale, cette "recréation mythologique des pays québécois » (Pelletier, 1996 : 151) semble pourtant vouée d'emblée à l'échec, puisque VLB décrète lui-même que « dans notre ici, pas de mythe et pas d'apparente possibilité d'en créer » (Beaulieu, 1976 : 151). Ce serait l'absence d'historicité du Québec, suggère Pelletier, qui « expliquerait dans une large mesure l'impossibilité de produire un récit épique authentique, dont le fondement mythique et magique demeure à venir, relevant du même coup d'un futur improbable, d'une libération collective de plus en plus problématique aux yeux de l'écrivain » (Pelletier, 2010 : 20). À la diffıculté de rappeler et 
de relater une histoire, qui est perçue par VLB comme une consternante suite de défaites, s'ajoute aussi l'absence d'une figure héroïque qui puisse concentrer les valeurs de la communauté.

Le héros épique, suggère Lukács dans sa Théorie du roman, est un sujet collectif qui n'agit jamais en tant qu'individu, mais incarne plutôt le destin d'une collectivité (Lukács, 1971 : 60-61). Un tel héros ferait défaut dans l'imaginaire québécois, ce qui empêche même les plus grands écrivains de concrétiser l'épopée nationale, comme le déplore VLB, estimant qu'il ne manque au Ciel de Québec de Jacques Ferron que cet élément fondamental, le héros, pour que nous assistions à l'odyssée elle-même plutôt qu'à la seule naissance de l'odyssée (Pelletier, 2010 : 20). C'est l'absence de cette figure centrale du genre épique, que VLB impute à l'ensemble de la littérature québécoise, qui causerait son enlisement, sa petite envergure, son incapacité à s'inscrire fortement dans la littérature mondiale. Nous verrons plus loin comment La Grande Tribu s'offre à pallier ce manque et à dénicher un héros à la hauteur dans le désastre de l'histoire de « [c] pays qui se niait pour ne pas produire de héros » (Beaulieu, 1976 : 148).

\section{LES LIBÉRATEURS / LES LÉSIONNAIRES}

Devant l'impossibilité de produire un récit épique cohérent, VLB livre l'une des œuvres les plus surprenantes et déconcertantes qu'il ait jamais écrites. En effet, La Grande Tribu déstabilise surtout par sa charpente narrative double, qui divise l'œuvre en deux volets alternant tout au long de ses 876 pages. Dans la partie intitulée «Les Libérateurs », VLB dresse le portrait de plusieurs intellectuels et hommes politiques selon une approche biographique très librement orientée vers son propos. Ce volet historique, qui fait l'objet d'une narration à la troisième personne, présente une galerie de personnages marquants du xixe siècle qui semblent n'avoir comme seul point commun que l'action militante, ou, plus largement, le partage d'une vision nationaliste. Certaines figures apparaissent incontournables, comme le patriote québécois Louis-Joseph Papineau ainsi que le parlementaire irlandais Daniel O'Connell, promoteur d'un nationalisme non-violent qui défendit les droits des catholiques d'Irlande et que VLB dépeint par ailleurs comme un réformisme prudent; Simon Bolivar, alias « Le Libertador », icône politique et militaire de l'émancipation des colonies espagnoles d'Amérique du Sud; ou encore Abraham Lincoln, président américain associé à l'abolition de l'esclavage aux États-Unis. En revanche, le choix d'autres noms plus problématiques, épinglés sur ce tableau d'honneur, déroute quelque peu. Songeons notamment à Charles Chiniquy, ancien prêtre catholique canadien reconverti en gourou fanatique, dont les frasques le propulsèrent au centre de nombreuses controverses aux États-Unis. Il obtient sa place sur le mur des révolutionnaires parce qu'il perturbera, selon les dires de VLB, pendant plus de 50 ans, la tranquillité de l'Église de Rome et sera «le plus extravagant de ses rebelles » (Beaulieu, $2008: 41$ ). Le cas du chinois Hong-Sieou-Tsiuan, chef de guerre et mystique fondamentaliste, laisse aussi perplexe : " [c]e sera de tous les rebelles du xixe siècle le plus étrange, le plus fascinant et le plus sanguinaire : au moins vingt millions de Chinois, à cause de lui, périront de mort violente dans une guerre qui durera dix ans comme celle de Troie » (Beaulieu, 2008 : 48). De ces derniers cas de figure se dégage un trait non négligeable : la violence, lorsqu'elle accompagne l'acte révolutionnaire, n'est pas réprouvée, car elle peut s'avérer nécessaire, voire inévitable à la cause. 
Ainsi, ce volet historique se destine, semble-t-il, à proposer plusieurs modèles de « héros » révolutionnaires en insistant sur la radicalité de leurs démarches lorsqu'elles s'y prêtent. Dans cette galerie de personnalités, la figure de Louis-Joseph Papineau occupe une place de choix, même si le sous-titre de l'œuvre suggère qu'une faute lui incombe, celle de s'être amendé auprès de l'occupant anglais, d'avoir renié les valeurs collectives et préféré l'exil à la potence. Dans son portrait, qui inaugure la section "Les Libérateurs ", le patriote québécois y est tout de même décrit comme un rebelle et comme « le Père de la nation, celui qui mettra fin à la traversée du désert à un peuple opprimé et lui fera atteindre la Terre promise, celle de l'indépendance, de la liberté et de l'égalité et de la fraternité » (Beaulieu, 2008 : 182). Toutefois, VLB l'accuse aussi d'être un réformiste frileux, plutôt conciliant avec l'envahisseur, répugnant la lutte sur le terrain.

Aux «Libérateurs » répondent « Les Lésionnaires », le deuxième volet de La Grande Tribu dans lequel le récit est pris en charge par Habaquq Cauchon, narrateur délirant détenu dans l'asile du docteur Avincenne pour subir une cure contre son « hystérie historique », trouble nerveux qui le pousse à fouiller compulsivement dans sa mémoire à la recherche de ses ancêtres. Le diagnostic du médecin sur l'origine du mal dont souffre Habaquq révèle « que c'est la faute à Papineau, que mon passé est plein de cette faute-là, que c'est pareil pour mon présent et que ça ne sera pas mieux dans mon avenir » (Beaulieu, 2008 : 63). Dans cette partie, le nom de Papineau est d'ailleurs cité à plusieurs reprises, notamment sous la forme d'une chanson' qui célèbre à la fois la gloire et les échecs de la nation canadienne-française, et dont les couplets sont scandés tout au long de l'œuvre

\section{Si les Kebekois si mous}

Ont toujours peur des loups-garous,

Si ne sentant plus leur importance,

Ils cauchemardent l'indépendance,

C'est la faute à Papineau,

C'est la faute, faute, faute,

\section{C'est la faute à Papineau!}

(Beaulieu, 2008: 545)²

La figure de Louis-Joseph Papineau joue ainsi un rôle ambigu, récupérée par le docteur Avincenne, qui s'en sert à la fois pour récuser le comportement de son patient et lui indiquer la bonne façon d'agir : "Cet homme-là - enseigne-t-il à Habaquq - a été rebelle alors que ce n'était pas le temps de l'être, [et il] s'est obstiné à le rester au point qu'il a dû s'exiler pour qu'on ne lui coupe pas la tête » (Beaulieu, 2008 : 445). Dans une entrevue au Devoir parue en février 2008, VLB explique : «Papineau a vraiment été le chef politique québécois qui a essayé d'aller le plus loin ... . Celui aussi qui a manqué son coup. On dirait que, depuis, tous ceux qui ont suivi n'ont fait que répéter ce grand ratage. Pour moi, à un certain niveau, le père fondateur du Québec - mais qui ne l'a pas été -, c'est vraiment Papineau » (Desmeules, 2008). Or, puisque Louis-Joseph Papineau a « manqué son coup » et que les chefs politiques qui lui ont succédé n'ont pas fait mieux, c'est dans le domaine

1. Sans doute inspirée de la chanson de Gavroche dans Les Misérables: « Je suis tombé par terre / C'est la faute à Voltaire / Je suis tombé dans le ruisseau / C'est la faute à Rousseau / Je suis tombé sous le tirant d'eau, c'est la faute, faute, faute / C'est la faute à Papineau » (Beaulieu, $2008: 230$ ).

2. Alors que cent pages plus tôt, un autre couplet de la chanson renverse le sens des paroles pour dire exactement le contraire. 
artistique que La Grande Tribu va chercher et élire son « héros » révolutionnaire : il s'agit du personnage de « l'orignal épormyable », une figure qui matérialise la présence surplombante dans l'œuvre de Gauvreau.

Personnage éponyme tiré d'une pièce de Gauvreau, La charge de l'orignal épormyable, il assume dans La Grande Tribu le rôle d'un héros qui traîne de lourdes responsabilités : libérer le « Kebec » de son aliénation culturelle, là où Louis-Joseph Papineau a échoué à le faire sur le plan politique. La représentation de Gauvreau dans La Grande Tribu reprend certains éléments véhiculés par l'histoire littéraire qui ont concouru à faire de lui un marginal incompris par ses contemporains. Seulement, cette image procède aussi d'une réinterprétation : remodelant la figure du poète martyr méprisé par sa société, VLB en forge un mythe nouveau, délibérément positif : celui du poète rebelle et révolutionnaire. Je me réfère ici aux conclusions de l'article de Sophie Dubois, «La grande tribu : c'est la faute à Gauvreau ou comme un orignal dans un jeu de quilles », qui traite de la multitude d'emprunts aux pièces de théâtre et à la poésie de Gauvreau recopiés - et même modifiés - au cœur de La Grande Tribu. Ce travail de réécriture opéré par la manipulation de l'intertexte gauvrien s'inscrit plus largement dans une entreprise d'appropriation totale de Claude Gauvreau au profit d'une vision sublimée, magnifiée du poète et de son héritage, laquelle met au jour le dessein derrière sa présence dans La Grande Tribu : proposer un modèle héroïque apte à guider le Québec vers sa révolution. Repiqués dans La Grande Tribu, les éléments tirés de l'œuvre gauvrienne convergent pour créer une image glorifiée du poète qui se négocie en fonction de l'autre mythe mis en présence dans l'œuvre, celui des «fondements hystériques du Québec » (Beaulieu, 1995 : 11). Selon Sophie Dubois, en faisant côtoyer le mythe de l'origine de la nation canadienne-française - perçu comme une succession de revers dont la révolte avortée des Patriotes en constitue l'apogée négative avec le mythe de Gauvreau, VLB lui fait porter le poids de siècles d'aliénation et l'élève au rang de rédempteur « venu venger, grâce à son verbe sacré, les injustices subies au cours de l'histoire par le peuple québécois » (Dubois, $2011:$ 75).

De fait, VLB trouve dans la poésie de l'orignal épormyable l'expression d'une violence libératrice qui sied à son propos. La description qui est faite de ce personnage insiste sur la force de son discours poétique et, surtout, sur son tempérament agressif :

Comme Émile Nelligan, l'orignal épormyable est aussi poète, du genre très claironnant et vociférateur, ce qui n'a pas fait l'affaire de grand-monde à cause que ça se claironne et vociférait dans une langue pour ainsi dire secrète, parfois rauque comme l'est l'allemande, parfois gutturale comme l'est l'irlandaise, et parfois punique comme l'est la romaine. Si un éléphant ça trompe énormément, l'orignal épormyable est porteur d'autrement plus de violence, il est fait pour charger, et sans qu'on ait besoin de le provoquer. . . Un poète rebelle, passe encore. Mais un poète révolutionnaire, qu'en faire quand ça ne cesse pas de hurler comme les mille et un gueulards du Saint-Maurice, et dans une langue autre que celle de la sagesse des nations? (Beaulieu, 2008: 71)

Ainsi, VLB fait de l'orignal épormyable un poète rebelle et révolutionnaire, mais « qui ne s'amendera pas comme Louis-Joseph Papineau l'a fait » (Beaulieu, 2008 : 71). II l'inscrit dans une généalogie mythique qui se revendique à la fois de Nelligan, lui aussi poète exceptionnel et marqué par le stigmate de la défaite, et des Patriotes, insurgés discrédités par l'histoire. 
Soulignant le ton « vociférant » sur lequel se déclame cette poésie, dans une «langue autre que celle de la sagesse des nations », VLB suggère son potentiel contestataire et la fureur qu'elle recèle. II s'agit d'une fureur expulsée par la violence dont l'orignal épormyable est porteur, celui-ci étant «fait pour charger, et sans qu'on ait besoin de le provoquer » (Beaulieu, 2008 : 71). Pourtant, peu importe son motif ou contre qui elle est dirigée, cette violence déployée par l'orignal épormyable est légitimée par le récit, qui lui octroie une valeur positive. Symbole d'un mouvement révolutionnaire qui s'ébranle et qui s'arroge le droit de dépasser les limites pour qu'un changement véritable s'opère, elle est aussi la seule réplique possible contre des bourreaux qui veulent étouffer sa force poétique.

Car si le texte insiste sur la puissance brute et imprévisible de ce personnage, c'est sa poésie qui constitue néanmoins la véritable arme révolutionnaire du récit. L'orignal épormyable est présenté comme « le plus colossal révolutionnaire de nos poètes » (Beaulieu, 2008 : 789), le seul apte à décrire « l'univers de plus en plus surréel » (Beaulieu, 2008 : 298) dans lequel les personnages vivent grâce à un nouveau mode d'expression poétique, l'exploréen, reformulé en « hyberboréen » dans La Grande Tribu. Or, c'est précisément ce langage obscur, jugé subversif par une société qui n'est pas encore prête à le recevoir, qui condamne l'orignal épormyable à l'asile psychiatrique, où le docteur Avincenne tente de le « guérir » par des méthodes de choc. Cette poésie automatiste, fondée sur le primat du son sur le sens, peut certes dérouter le lecteur, qui se retrouve face à une logorrhée de mots créés de toutes pièces ou associés les uns aux autres par une logique désarticulée. Dans une note provisoire sur les $\mathbb{E} u v r e s$ créatrices complètes de Gauvreau, Pierre Nepveu souligne:

Le langage de Gauvreau ne nous parle pas, [il] ignore presque systématiquement les lois de la communication, de l'empathie et du lyrisme. Cette ignorance, il en fait son unique loi, il la cultive avec un acharnement et une ténacité qui tiennent de la pathologie. D'entrée de jeu, nous lecteurs sommes exclus, indésirables; ce texte ne nous aime pas, sinon comme voyeurs. Et ce que nous voyons ressemble étrangement à un immense et déraisonné plaisir solitaire. (Nepveu, 1977 : 17)

De façon un peu plus nuancée, on peut concevoir que le langage de Gauvreau tente de parler, de s'adresser à un lecteur, qui, échouant à le comprendre, se sent nécessairement exclu de la communication. Nepveu conclut que «l'automatisme de Gauvreau opte décidément pour le gaspillage », arguant que «le radicalisme de [son] écriture » se complaît dans le « refus de toute concession à la communication » (Nepveu, 1977 : 18). S'il est vrai que la poésie gauvrienne refuse toute forme de compromis pour faciliter l'entendement, il paraît un peu fort de parler du « gaspillage » de cette somme de mots qui ne passe pas l'épreuve du sens.

Même si elle demeure diffıcile d'accès, la poésie de Gauvreau interpelle brusquement son lecteur par sa pratique de l'invective, exige son entière attention et, surtout, l'oblige à la confrontation. Parce qu'elle est dans La Grande Tribu une poésie déclamée ou plutôt bramée de vive voix par l'orignal épormyable, elle peut être vue comme une tentative répétée, mais non concluante, d'interagir avec son public. En effet, la poésie de l'orignal épormyable, largement conspuée au cœur du récit de La Grande Tribu, ne parvient pas à emporter l'adhésion du public et à souder autour d'elle l'unité et l'identité nationales. Toutefois, elle trouve un admirateur en la personne d'Habaquq Cauchon, même si celui-ci admet ne pas la saisir : " J'ai souvent entendu l'orignal épormyable quand il bramait ainsi en langage exploréen, mais je n'y ai jamais rien compris : tous ces mots bizarres asso- 
ciés par étrangeté dans des phrases que domine l'onomatopée, à quels sens se nourrissent-ils? » (Beaulieu, 2008 : 763).

La réponse à cette question, comme le supposent les extraits de la poésie et du théâtre de Gauvreau reproduits dans La Grande Tribu, s'explique par une soif de liberté et de justice qui finit par s'incarner concrètement dans les actions des personnages, alors que Habaquq Cauchon et l'orignal épormyable sont enrôlés dans une cellule terroriste dissimulant ses activités sous l'enseigne du Parti des lésions, qui se veut à la fois une université populaire et un mouvement d'intervention politique. Ils tentent de faire entendre raison au gouvernement, qui vient de nommer le docteur Avincenne, leur tortionnaire, au poste de ministre de la Santé. En conférence de presse, l'orignal brame son poème "Ode à l'ennemi », une charge virulente contre l'oppresseur qui exprime l'idée que la liberté n'est possible que par la destruction, sans toutefois réussir à attirer l'attention des folliculaires et des députés présents. Si cette tentative de rallier le gouvernement à sa cause par la parole poétique se solde par un échec en raison du cynisme et du despotisme des autorités, la réplique est dure : I'orignal épormyable, Habaquq Cauchon et Bowling Jack, chef du Parti des lésions, s'introduisent dans le « Parlement de Kebec » en fauteuils roulants.

Munis de bombes, ils menacent de tout faire exploser si le gouvernement ne vote pas à l'unanimité la déclaration d'indépendance de la nation québécoise. Ils se défendent de l'épithète « terroriste » qu'on leur adresse : «On appelle terroristes les patriotes et les rebelles qui manquent leur coup ... Ceux qui triomphent, ce sont des libérateurs et ils ont tous leur place dans le grand cancer des nations. Est-ce utile que je vous en donne de probants exemples? » (Beaulieu, 2008 : 819). II semble que ces exemples soient déjà donnés dans la partie "Les Libérateurs » de La Grande Tribu : ces hommes qui ont réussi à conduire leur peuple à la libération et qui n'ont pas craint, pour réaliser ce projet, d'employer des méthodes un peu plus radicales, quitte à ce que le sang coule. En ce sens, la fin de l'œuvre condense cette phrase, prononcée dans Les oranges sont vertes, une pièce de Gauvreau jouée par les personnages de La Grande Tribu, alors que ceux-ci renoncent au bout du compte au pouvoir du Langage pour des moyens plus draconiens : « L'ère de la poésie est définitivement morte. Va lui succéder le temps du terrorisme » (Beaulieu, 2008 : 765).

Nous avons proposé de qualifier la démarche de VLB dans La Grande Tribu de «terroriste », parce que, favorisant une poésie radicale qui ne fait aucun compromis sur le sens et qui ne parvient pas à créer un dialogue, elle conclut à l'impuissance des mots à changer les choses et convoque de la sorte la nécessité d'une action d'éclat, quitte à devoir exhiber la menace de la violence.

II n'est pas anodin d'ailleurs de constater que les deux modèles héroïques qui sont opposés dans La Grande Tribu, soit Louis-Joseph Papineau et Claude Gauvreau, s'avèrent tous deux des figures d'orateurs : Papineau, celui qu'on surnomme Le Verbe, selon VLB, parce que la parole s'était réincarnée dans son corps, ce qui lui permet de briller dans les débats parlementaires, tandis que Gauvreau est présenté comme le plus grand des poètes, et surtout, comme un poète vociférant et claironnant. Mais alors que Gauvreau constitue le nouveau modèle révolutionnaire dans lequel VLB souhaiterait engager le Québec, la société du texte refuse de suivre l'orignal épormyable dans la surréalité qu'il propose et reste attachée à l'image de Papineau. Ce dernier a été le premier, dit VLB, à faire de l'idée d'indépendance le projet fondamental de sa vie, mais aussi celui qui a échoué à concrétiser ce projet, et dont l'échec conditionne désormais la destinée de ce «pays- 
pas-encore-pays », comme VLB le surnomme. Ce projet révolutionnaire semblait donc condamné d'avance parce que le Québec vit toujours dans l'attente de ce que l'écrivain appelle « les conditions gagnantes », ce qui constituerait en quelque sorte un dépassement de l'aliénation dans laquelle vivrait toujours la nation, ou, en d'autres mots, l'indépendance enfin gagnée.

La fin de La Grande Tribu ne révèle pas clairement si l'attaque kamikaze du Parti des lésions à I'Assemblée nationale réussit à persuader le gouvernement de déclarer cette indépendance, mais nous pouvons tout de même le supposer, du fait que deux des personnages principaux sont toujours en vie. Ce dénouement positif, qui semble annoncer que l'action terroriste ait porté fruit, se termine sur le retour du couplet de la chanson "C'est la faute à Papineau », cité précédemment. Seulement, les paroles sont retournées de sorte qu'elles signalent la victoire de l'indépendance et signent du même coup une certaine réconciliation avec le passé et avec Louis-Joseph Papineau. L'avenir ne semble désormais plus aussi sombre, et Habaquq Cauchon peut enfin conclure : «Nous n'avons plus hâte. Nous n'attendons plus. Nous avons entrepris » (Beaulieu, 2008 : 863). 
Beaulieu, Victor-Lévy. James Joyce, l'Irlande, le Québec, les mots. 2006. Montréal : Éditions du Boréal, 2010. Imprimé.

---. La Grande Tribu : c'est la faute à Papineau. Montréal : Éditions du Boréal, 2008. Imprimé.

---. Le Carnet de l'écrivain Faust. Montréal : Stanké, 1995. Imprimé.

---. N'évoque plus que le désenchantement de ta ténèbre, mon si pauvre Abel. Montréal : VLB éditeur, 1976. Imprimé.

Desmeules, Christian. «Entretien - C'est la faute à VLB. » Le Devoir. 23 février 2008. En ligne. 10 août 2012.

Dubois, Sophie. "La grande tribu : c'est la faute à Gauvreau ou comme un orignal dans un jeu de quilles. » Les Cahiers Victor-Lévy Beaulieu, vol. I : Actualités de Victor-Lévy Beaulieu. Québec : Éditions Nota Bene, 2011. 71-96. Imprimé.

Guy, Chantal. «L'ultimatum de VLB. » Vigile.net. 2 mars 2008. En ligne. 10 août 2012.

Lukács, Georg. La Théorie du roman. Paris: Gonthier, 1971. Imprimé.

Nepveu, Pierre. « Note provisoire sur les Euvres créatrices complètes. » Lettres québécoises : la revue de l'actualité littéraire 7 (1977) : 17-18. Imprimé.

Pelletier, Jacques. Croisements littéraires et politiques : écriture et émancipation. Écrits à contre-courant 3. Québec: Nota Bene, 2010. Imprimé.

---. L'Écriture mythologique : essai sur l'œuvre de Victor-Lévy Beaulieu. Québec : Nuit Blanche Éditeur, 1996. Imprimé. 